\title{
Evaluation of praecordial ST segment mapping as an index of infarct size in patients with acute myocardial infarction ${ }^{1}$
}

\author{
MICHITOSHI INOUE, MASATSUGU HORI, MASATAKE FUKUNAMI \\ MASAKATSU FUKUSHIMA, MICHIHIKO TADA, HIROSHI ABE, \\ TAKAZO MINAMINO, AND SUGAO FUKUI \\ From the First Department of Medicine, Osaka University Medical School, and Sakurabashi Watanabe \\ Hospital
}

SUMMARY We evaluated the usefulness and limitations of praecordial ST segment mapping as a clinical means of assessing the size of acute myocardial infarction in 14 patients with anterior myocardial infarction and 13 patients with inferior myocardial infarction. $\Sigma S T$, the sum of ST segment elevations, and nST, the number of leads showing ST segment elevation, were obtained from serial electrocardiograms recorded through 39 praecordial leads. The infarct size and period of the evolution of myocardial infarction were estimated respectively from the total creatine kinase (CK) released and the serial changes of the $\mathrm{CK}$ releasing rate. $\Sigma S T$ and $\mathrm{nST}$ obtained at the time when the CK release had ceased correlated closely with the total CK released. Peak $\Sigma S T$ and nST, and values 48 hours after the onset of myocardial infarction, also correlated well with the total CK released; but those on admission or 12 hours after the onset correlated poorly.

These results suggest that $\Sigma S T$ and nST at the end of evolution of myocardial infarction or 48 hours after the onset may be two useful indices for the assessment of infarct size in patients with either anterior or inferior myocardial infarction.

Many attempts (Maroko et al., 1972; Norris et al., 1975; Henning et al., 1978; Sharpe et al., 1978) have been made to assess infarct size in patients with acute myocardial infarction, because the extent of necrosis directly influences cardiac function and subsequent prognosis (Page et al., 1971; Sobel et al., 1972; Mathey et al., 1974; Hori et al., 1979). Maroko et al. (1971) reported a close correlation between the magnitude of epicardial ST segment elevation 15 minutes after experimental coronary occlusion in dogs, and infarct size as determined by loss of myocardial creatine kinase (CK) activity or as determined histologically 24 hours later. Their further study (Muller et al., 1975) also showed that in dogs the magnitude of epicardial ST segment elevation correlated well with that of praecordial ST segment elevation. Though it is now widely recognised that surface mapping of the electrocardiogram is useful in evaluating the effect of therapeutic interventions on the extent of infarc-

1 Supported by grants from the Ministry of Education, Science and Culture, the Ministry of Health and Welfare, and Japan Heart Foundation Research for 1979.

Received for publication 20 February 1979 tion, the usefulness of this technique in measuring human infarct size remains controversial (Reese et al., 1973; Norris et al., 1976; Thompson and Katavitis, 1976) perhaps because serial changes of ST segment elevation after the onset of infarction have been studied inadequately. In this study we attempted to investigate the serial changes in the praecordial ST segments to evaluate the feasibility of the surface mapping technique as an index of infarct size both in patients with anterior infarction and with inferior infarction.

\section{Subjects and methods}

Of 34 consecutive patients with acute myocardial infarction who were admitted to the Coronary Care Unit of Sakurabashi Watanabe Hospital within 12 hours after the onset of infarction, 27 were studied. We excluded two patients with previous myocardial infarction, three patients whose infarct size could not be assessed because of early death, and two patients with intraventricular conduction delay. None of the 27 patients in our series had pericarditis or a cerebrovascular accident which might influence 
ST segment changes. Fourteen patients had anterior infarction (13 men and one woman, ages 33 to 77, with a mean of 53 years). This group included one patient with lateral infarction and another with dominantly subendocardial infarction and only partial transmural anterior infarction; these two were excluded from the statistical analysis. Thirteen patients had inferior infarction (eight men and five women, ages 44 to 82 years, with a mean of 66 years). The diagnosis was made by a typical history, characteristic alterations of ST-T wave changes on the standard 12 lead electrocardiograms, and diagnostic rise and fall in serum enzymes (creatine kinase, $\alpha$-hydroxybutyric dehydrogenase, aspartate aminotransferase, and lactic dehydrogenase).

\section{(1) PRAECORDIAL ST SEGMENT MAPPING TECHNIQUE}

Electrocardiograms were recorded through unipolar leads ('V' lead) from 39 points, which were marked with an indelible pen so that all subsequent recordings were obtained from the same points. The 39 points comprised 21 points on the anterior chest and 18 points on the left lateral chest as shown in Fig. 1.

Electrocardiograms were recorded, using a thermal pen recorder on standard electrocardiographic paper running at $25 \mathrm{~mm} / \mathrm{s}$ with standardisation of $1 \mathrm{mV} / \mathrm{cm}$, on admission and 12, 24, 36, 48, 72 hours, and seven and 14 days after the onset of chest pain. Whenever possible, electrocardiograms were also obtained three and six hours after the onset of pain. The magnitude of ST segment elevation of recorded electrocardiograms was measured at the $\mathrm{J}$ point to the nearest $0.5 \mathrm{~mm}$.

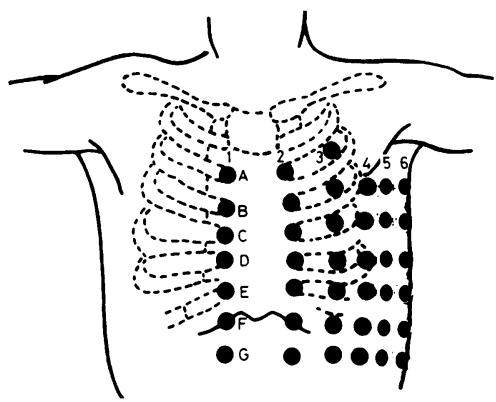

Fig. 1 The positions of 39 praecordial leads. Twenty-one points on the anterior chest were at the intersections of three imaginary vertical lines (1: right sternal border; 2: left sternal border; 3 : left mid-clavicular line) and seven horizontal lines ( $A-E$ : second to sixth intercostal space near the sternum; $F: 2.5 \mathrm{~cm}$ below $E ; G: 2.5 \mathrm{~cm}$ below $F)$. Eighteen points on the left lateral chest were at the intersections of three vertical lines (4: anterior axillary line; $5:$ mid-axillary line; $6:$ posterior axillary line) and six horizontal lines ( $B$ to $G$ as above).
The sum of ST segment elevations from all leads

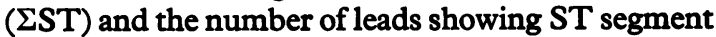
elevation of $0.5 \mathrm{~mm}$ or greater (nST) were calculated from each recording.

In order to evaluate the reproducibility of measured ST segment elevation, the praecordial ST segment mapping was recorded again one hour after we recorded the three-hour or the 48-hour electrocardiograms in 10 patients. Two cardiologists independently measured ST segment deviation in each electrocardiogram recorded from five patients to assess the variation among observers.

\section{(2) ASSESSMENT OF INFARCT SIZE BY TOTAL CK RELEASED ( $\Sigma \mathrm{CK}$ )}

Venous blood samples for CK determination were obtained at four-hour intervals from the time of admission until serum enzyme levels returned to normal. The appearance function of $\mathrm{CK}$ released from the infarcted myocardium was calculated by the method of Sobel et al. (1972) as modified by Norris et al. (1975) and used as a guide in assessing the time course of evolution of the infarct (Inoue et al., 1977). Total CK released ( $\Sigma C K$ ) was measured by integrating this appearance function and was used as an index of infarct size. Total CK released up to 24 and 48 hours after the onset, $\Sigma C_{24}$ or $\Sigma \mathrm{CK}_{48}$, respectively, were also measured in order to compare the change in $\Sigma S T$ from 24 to 48 hours. Though the evolution of the infarct was considered to cease at the time when the appearance function of $\mathrm{CK}$ released became zero $\mathrm{IU} / \mathrm{ml}$ per $\mathrm{h}$ (Inoue et al., 1977), the exact determination of this time was difficult because it could be influenced substantially by subtle alterations in the disappearance rate of serum $\mathrm{CK}$ activity. Therefore, in this study the time when the appearance function decreased to $5 \mathrm{IU} / \mathrm{ml}$ per $\mathrm{h}$ was regarded as the end point of evolution; this time is much less influenced by the alteration in the disappearance rate of serum CK activity. In practice, $\Sigma S T$ and $\mathrm{nST}$ at the end of evolution were obtained by linear interpolation of the two values which were measured at the closest points immediately before and after the time when the appearance function became $5 \mathrm{IU} / \mathrm{ml}$ per $\mathrm{h}$ or less.

\section{Results}

(A) EVALUATION OF ST SEGMENT MAPPING

AS AN INDEX OF INFARCT SIZE

(1) Serial changes in $\Sigma S T$ and $n S T$

$\Sigma S T$ and $\mathrm{nST}$ were obtained on admission and $12,24,36,48$, and 72 hours, and on the seventh and 14th day after the onset in all patients studied. $\Sigma S T$ and $n S T$ were also obtained three and six 
hours after the onset in six and 17 patients, respectively. Fig. 2 and 3 show the serial changes in $\Sigma S T$ and $\mathrm{nST}$ in patients with anterior and inferior infarction. The data three hours after the onset in patients with inferior infarction are not depicted in Fig. 3 since they were obtained in only two patients. Serial changes in $\Sigma S T$, nST, and the appearance function of $\mathrm{CK}$ in two representative cases are shown on the same time scale in Fig. 4 and 5.

In one case with anterior infarction shown in Fig. 4, $\Sigma S T$ and $\mathrm{nST}$ increased to their peaks 48 hours and 24 hours after the onset, respectively, and then decreased gradually. In contrast, another

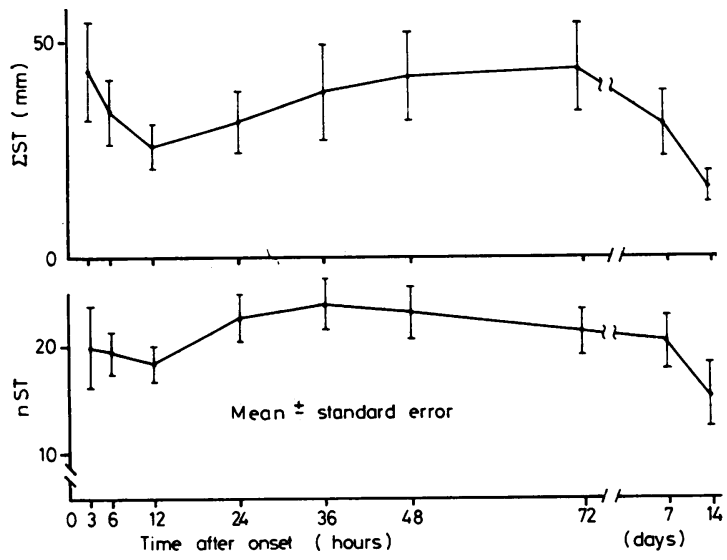

Fig. 2 The time course of mean values of $\Sigma S T$ (upper panel) and nST (lower panel) after the onset in 12 patients with anterior infarction. $\Sigma S T$ and $n S T$ three and six hours after the onset were obtained in four and nine patients, respectively.
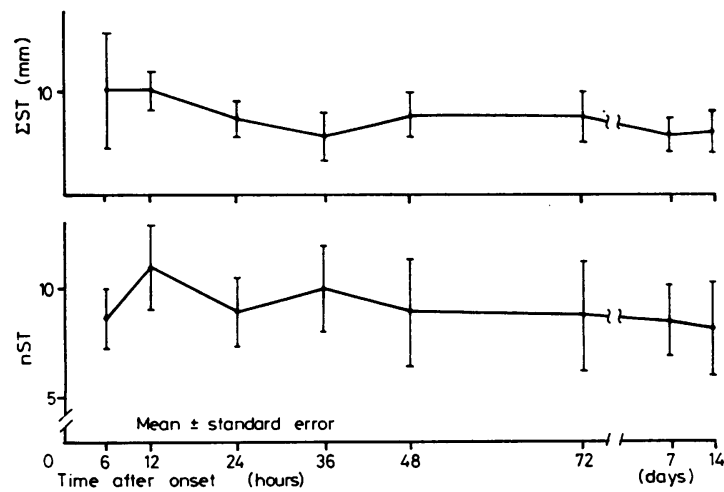

Fig. 3 The time course of mean values of $\Sigma S T$ (upper panel) and $n S T$ (lower panel) after the onset in 13 patients with inferior infarction. These indices six hours after the onset were obtained in eight patients. The data three hours after the onset were not depicted since they were obtained in only two patients.
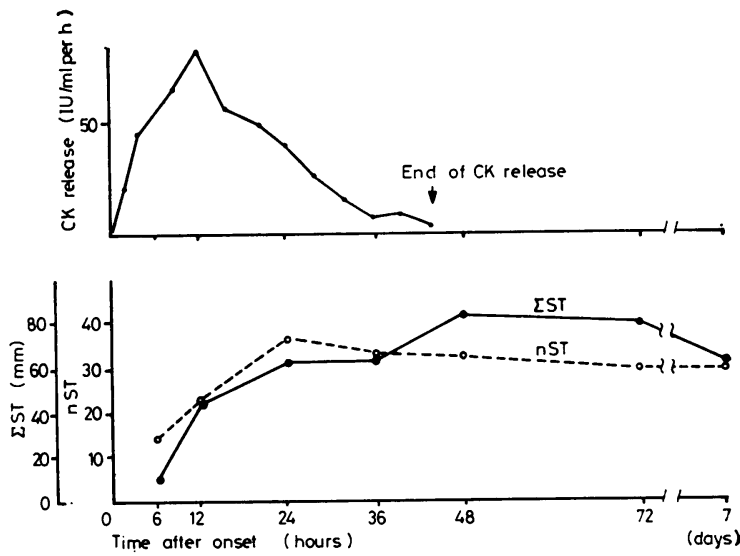

Fig. 4 Serial changes in $C K$ release per hour (upper panel), $\Sigma S T$ and $n S T$ (lower panel) in a representative case with anterior infarction.
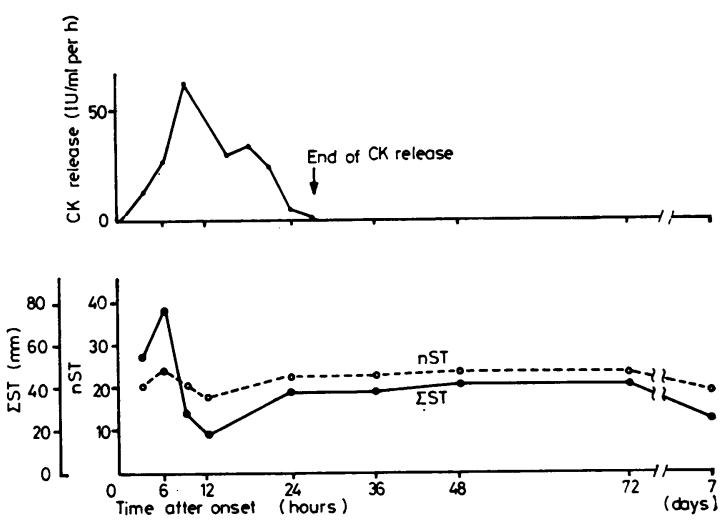

Fig. 5 Serial changes in $C K$ release per hour (upper panel), $\Sigma S T$ and $n S T$ (lower panel) in a representative case with anterior infarction.

case with anterior infarction showed a typical biphasic pattern of $\Sigma S T$ and $\mathrm{nST}$ as shown in Fig. 5; both indices showed a transient decrease between six and 12 hours after the onset of infarction and then increased again without any important changes in blood pressure, heart rate, or symptoms. Such a biphasic pattern was observed in six out of 12 patients $(50 \%)$ with anterior infarction and eight out of 13 patients $(62 \%)$ with inferior infarction. It was of great interest, however, that in almost all patients both $\Sigma S T$ and nST reached a somewhat stable state at the end of evolution of infarction judged from the appearance function of CK released (see Methods) and showed a gradual decrease thereafter, as shown in Fig. 4 and 5.

(2) $\Sigma S T$ and $n S T$ at end of evolution of infarction The mean values of $\Sigma S T$ and $n S T$ at the end of 
evolution of infarction (henceforth designated as $\Sigma S T_{\text {end }}$ and $n S T_{\text {end, }}$ respectively) and the duration of evolution (the duration of CK release) are shown in Table 1. It was seen that $\Sigma S T_{\text {end }}$ andn $S T_{\text {end }}$ correlated closely with the total $\mathrm{CK}$ released, which represents the infarct size in 12 patients with

Table 1 Mean values of $\Sigma S T$ and $n S T$ at end of evolution of infarct and duration of evolution in patients with anterior and inferior infarction

\begin{tabular}{|c|c|c|c|c|}
\hline & $\begin{array}{l}\text { Number } \\
\text { of cases }\end{array}$ & $\begin{array}{l}\text { SST end } \\
(S E M) \\
(m m)\end{array}$ & $\begin{array}{l}n S T_{\text {end }} \\
(S E M)\end{array}$ & $\begin{array}{l}\text { Duration of } \\
\text { evolution } \\
(S E M)(h)\end{array}$ \\
\hline $\begin{array}{l}\text { Anterior myocardial } \\
\text { infarction }\end{array}$ & $\mathrm{n}=14$ & $35 \cdot 3=7 \cdot 3$ & $21.5=2.2$ & $38 \cdot 7=4$ \\
\hline $\begin{array}{l}\text { Inferior myocardial } \\
\text { infarction }\end{array}$ & $n=13$ & $10 \cdot 2: 0$ & 11.01 .6 & $41.0 \div 3 \cdot 7$ \\
\hline
\end{tabular}

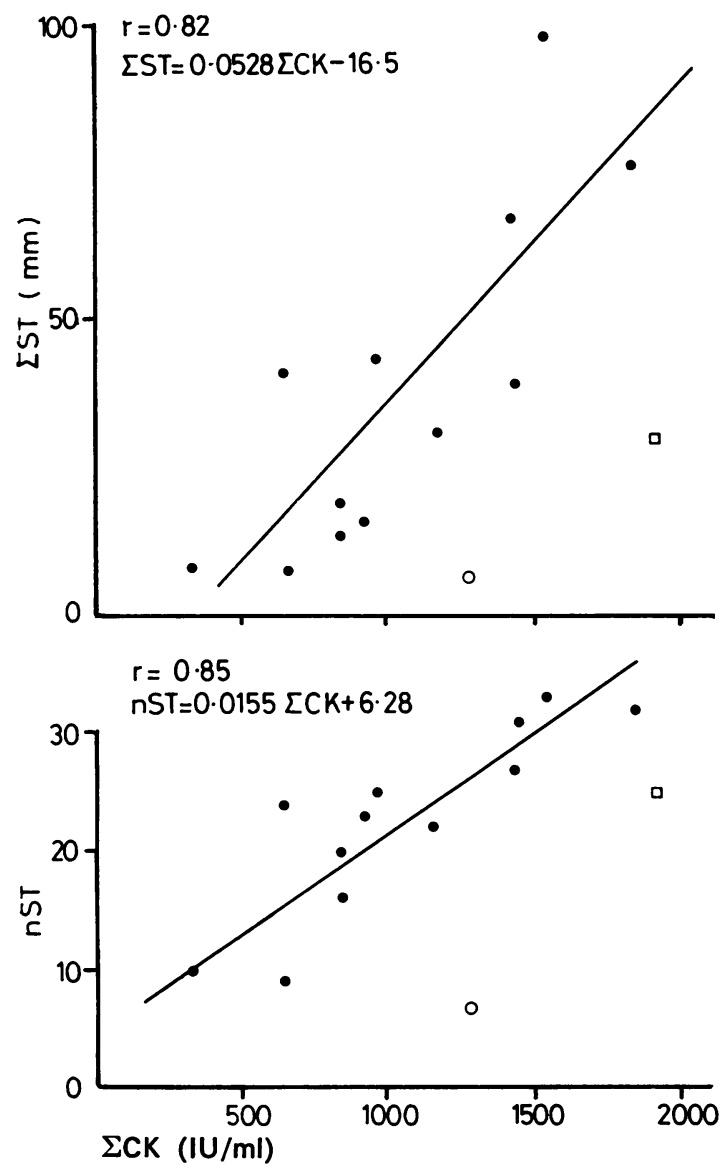

Fig. 6 Relations between $\Sigma S T$ (upper panel), $n S T$ (lower panel), and the total $C K$ released $(\Sigma C K)$ at the end of evolution of anterior infarction. $\square:$ a case with ateral infarction; $O:$ a case with subendocardial infarction and partial transmural anterior infarction. anterior infarction ( $\Sigma S T$ : $r=0.82, P<0.01$;nST: $r=0.85, P<0.01$, see Fig. 6). Values from two patients who were excluded from the statistical analysis (one with lateral infarction and another with subendocardial infarction and only partial transmural anterior infarction) were also plotted in Fig. 6; they were far below the regression line, indicating that these $\Sigma S T$ and $\mathrm{nST}$ were disproportionately too small in comparison with the infarct size estimated enzymatically. In 13 patients with inferior infarction there was also a significant correlation between $\Sigma S T_{\text {end }}$ and $\mathrm{nST}_{\text {end }}$ and infarct size $(\Sigma C K)\left(\Sigma S T_{\text {end }}: \mathrm{r}=0.84, \mathrm{P}<0.01 ; \mathrm{nST}_{\text {end }}\right.$ : $r=0.72, P<0.01$, see Fig. 7). These results strongly suggest that $\Sigma S T$ and $n S T$ at the end of the evolution of infarction directly reflect infarct size in both anterior infarction and inferior infarction, but not for lateral or subendocardial infarction.

(3) $\Sigma S T$ and nST 48 hours after onset of infarction Since in 80 per cent of all patients the evolution of infarction had ceased within 48 hours after the onset of chest pain, the relation between $\Sigma S T$ and nST 48 hours after the onset (designated as $\Sigma \boldsymbol{\Sigma T}_{48}$ and $\mathrm{nST}_{48}$, respectively) and infarct size ( $\left.\Sigma \mathrm{CK}\right)$
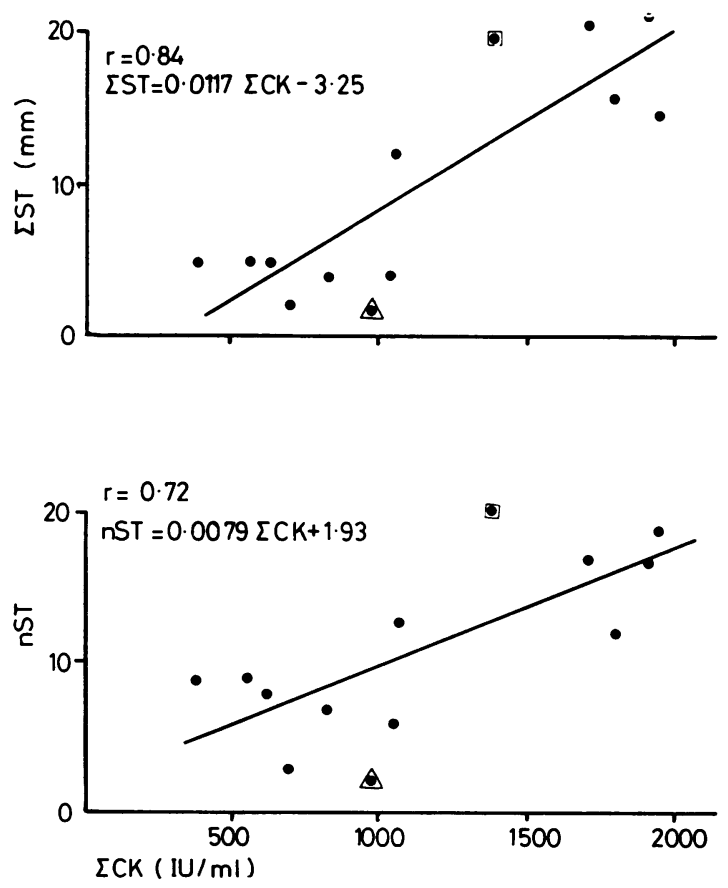

Fig. 7 Relations between $\Sigma S T$ (upper panel), $n S T$ (lower panel), and the total $C K$ released ( $\Sigma C K$ ) at the end of evolution of inferior infarction. $\square:$ a case with inferoseptal infarction; 1 : case with inferoposterior infarction. 
was also investigated. We found that $\Sigma \mathrm{ST}_{48}$ and $\mathrm{nST}_{48}$ also correlated significantly with $\Sigma \mathrm{CK}$ in both anterior infarction and inferior infarction; $r=0.72(P<0.01)$ for $\Sigma S T_{48}$ and $r=0.77(P<0.01)$ for $\mathrm{nST}_{48}$ in anterior infarction and $\mathrm{r}=0.86$ $(\mathrm{P}<0.01)$ for $\Sigma S T_{48}$ and $r=0.66(P<0.01)$ for $n S T_{48}$ in inferior infarction (see Table 2).

Table 2 Correlations between $\Sigma S T$ and/or $n S T$ and total $C K$ released ( $\Sigma C K)$ at various time intervals after onset of infarction

\begin{tabular}{|c|c|c|c|c|}
\hline & \multicolumn{2}{|c|}{ Anterior infarction } & \multicolumn{2}{|c|}{ Inferior infarction } \\
\hline & $\therefore S T$ & $n S T$ & $\because S T$ & $n S T$ \\
\hline 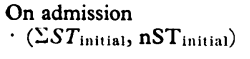 & 0.43 & $0 \cdot 67^{\star}$ & $0.63^{\star}$ & 0.53 \\
\hline $\begin{array}{l}\text { At } 12 \mathrm{~h} \text { after onset } \\
\left(\Sigma \mathrm{ST}_{12}, \mathrm{nST}_{12}\right)\end{array}$ & 0.58 & 0.58 & $0.74 \dagger$ & 0.55 \\
\hline $\begin{array}{l}\text { At } 48 \mathrm{~h} \text { after onset } \\
\left(\because \mathrm{ST}_{48}, \mathrm{nST}_{48}\right)\end{array}$ & $0.72 \dagger$ & $0.77 \dagger$ & $0.86 \dagger$ & $0.66 \dagger$ \\
\hline $\begin{array}{l}\text { At peak time } \\
\left(\mathrm{ST}_{\max }, \mathrm{nST}_{\max }\right)\end{array}$ & $0.76 \dagger$ & $0.86 \dagger$ & $0.79 \dagger$ & $0.68 \dagger$ \\
\hline $\begin{array}{l}\text { At end of evolution } \\
\left(\Xi \mathrm{ST}_{\text {end }}, \mathrm{nST}_{\text {end }}\right)\end{array}$ & $0.82 \dagger$ & $0.85 t$ & $0.84 \dagger$ & $0.72 \dagger$ \\
\hline
\end{tabular}

\section{(4) $\Sigma S T$ and $n S T$ at their peaks}

In our series maximal $\Sigma S T$ and nST were obtained at $38.3 \pm 7.0$ (SEM) hours and $33.3 \pm 5.8$ (SEM) hours, respectively, in patients with anterior infarction, and $31.9 \pm 6.3$ (SEM) hours and 25.9 \pm 4.3 (SEM) hours, respectively, in patients with inferior infarction. The maximal $\Sigma S T$ and $n S T$ (henceforth designated as $\Sigma S \mathrm{~T}_{\max }$ and $\mathrm{nST} \mathrm{T}_{\max }$ ) also correlated significantly with the infarct size $(\Sigma C K): r=0.76$ $(P<0.01)$ for $\Sigma S T_{\max }$ and $r=0.86(P<0.01)$ for nST $_{\max }$ in anterior infarction, and $\mathrm{r}=0.79(\mathrm{P}<0.01)$ for $\Sigma S T_{\max }$ and $\mathrm{r}=0.68(\mathrm{P}<0.01)$ for $\mathrm{nST}_{\max }$ in inferior infarction.

\section{(5) $\Sigma S T$ and $n S T$ on admission and 12 hours after onset of infarction}

$\Sigma S T$ and nST on admission ( $\Sigma S T_{\text {initial }}$ and $\left.n S T_{\text {initial }}\right)$ and 12 hours after the onset $\left(\Sigma S T_{12}\right.$ and $\mathrm{nST}_{12}$ ) were found to be poorly correlated with total CK released (especially $\Sigma S T_{\text {initial }}$ ) in anterior infarction $(r=0.43, P>0.05)$ and $n S T$ initial in inferior infarction $(r=0.53, P>0.05$, see Table 2$)$. These results strongly suggest that $\Sigma S T$ and $\mathrm{nST}$ during the early phase of the progression of infarction do not directly reflect the extent and severity of tissue necrosis of the myocardium.

(6) Detection of extension of infarction by serial change of $\Sigma S T$ or $n S T$

To investigate whether the serial change of $\Sigma S T$ or nST can directly reflect the instantaneous evolution of infarct, the change of $\Sigma S T$ or $\mathrm{nST}$ between 24 and 48 hours after the onset was compared with the amount of increase in CK released during this interval $\left(\Sigma \mathrm{CK}_{24}\right.$ to $\left.\Sigma \mathrm{CK}_{48}\right)$. In five of 11 patients $(45.5 \%)$ with anterior infarction and nine of 13 patients $(69 \%)$ with inferior infarction, $\Sigma S T$ increased with the increase in $\Sigma C K$, shown by arrows in the right upper part of Fig. 8. One patient with anterior infarction was excluded because the electrocardiogram recorded 24 hours after the onset showed transient right bundle-branch block. In this figure, however, some arrows point downwards, indicating that $\Sigma S T$ decreased from 24 to 48 hours after the onset of infarction. This suggests that $\Sigma S T$ decreases in some patients despite the evolution of infarct. This was also true of nST.

In summary, our results indicate that the infarct size in patients both with anterior infarction and with inferior infarction may be estimated by $\Sigma S T$ and nST obtained from the praecordial electrocardiographic mapping at the time when the
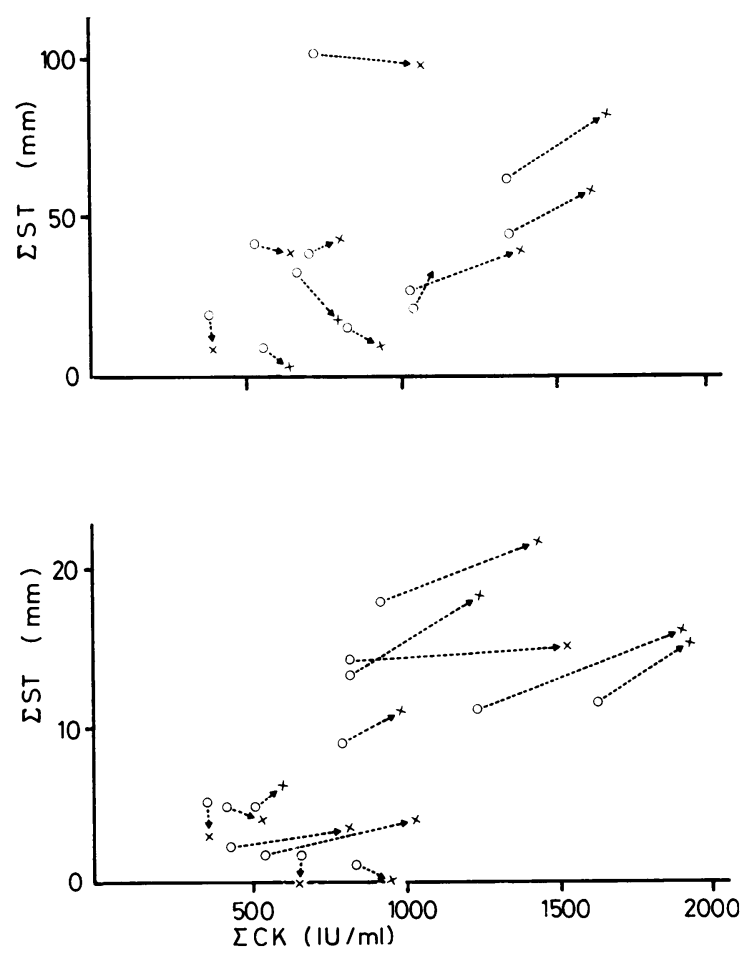

Fig. 8 Individual changes in $\Sigma S T$ relating to the changes in $\Sigma C K$ till the corresponding time to $\Sigma S T$ in 11 patients with anterior infarction (upper panel) and in 13 patients with inferior infarction (lower panel). Open circle and cross mark represent the data obtained 24 and 48 hours after the onset, respectively. Dashed arrow represents the direction of change from 24 to 48 hours after the onset. 
evolution of infarct has ceased ( $\Sigma S T_{\text {end }}$ and $\mathrm{nST}_{\text {end }}$ ) or 48 hours after the onset, when the evolution is considered to have been completed in most of the cases $\left(\Sigma S T_{48}\right.$ and $\left.n S T_{48}\right) . \Sigma S T$ and $n S T$ at these times after the onset closely correlated with infarct size ( $\Sigma C K)$, probably because ST segment elevation becomes stable after evolution of the infarct has ceased, and the ischaemic injury zone would be proportional to the extent of tissue necrosis at this stage of the disease. In contrast, however, at the early stage during the evolution of the infarct, praecordial ST segment elevation does not reflect the infarct size. Thus, $\Sigma S T$ and nST 12 and 24 hours after the onset correlated poorly with $\Sigma$ CK, probably because the surrounding ischaemic zone is labile and greatly influenced by small alterations in coronary blood supply and oxygen demand during evolution of the infarct.

\section{(B) REPRODUCIBILITY OF ST SEGMENT} MAPPING TECHNIQUE

The variation in measurement of ST segment displacement was $7 \cdot 6 \pm 8 \cdot 3$ per cent (mean $\pm S D$ ) in 25 recordings obtained from five patients, indicating that errors from this source are small. Though the hour-to-hour variation about three hours after the onset was $26.7 \pm 8.9$ per cent (mean $\pm S D$ ) in five patients, the variation about 48 hours after onset was $3.5 \pm 2.6$ per cent (mean $\pm S D$ ) in five patients.

These results suggest that $\Sigma S T$ obtained about 48 hours after the onset is stable enough to be meaningful.

\section{Discussion}

In this study we evaluated the validity of praecordial ST segment mapping and also its limitation as an index of infarct size in patients both with anterior infarction and with inferior infarction. The sum of

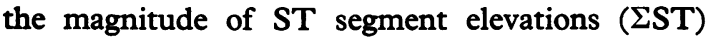
and the total number of leads showing ST segment elevation (nST) during the early phase of infarction, within 12 hours after the onset, did not accurately reflect the extent of infarction. However, $\Sigma S T$ and/ or $\mathrm{nST}$ measured at the time when the evolution of infarct had ceased or the maximal $\Sigma S T$ and/or nST did closely correlate with infarct size. This method may be useful in patients with inferior as well as anterior infarction.

Since Maroko et al. (1971) established epicardial ST segment mapping as a method for the assessment of infarct size, it has been predicted that praecordial ST segment mapping would also be useful clinically to evaluate the effect of therapeutic interventions during the course of acute myocardial infarction and also to detect the extension of infarction. However, the validity of quantification of praecordial ST segment elevation for the assessment of infarct size has remained controversial. Norris et al. (1976) reported that there was no significant correlation between the peak value of $\Sigma S T$ and the infarct size estimated by the same method in our study. Thompson and Katavitis (1976) also showed the clinical limitation of this method; only a rough correlation $(r=0.48, n=12)$ was found between ST segment elevation and infarct size $(\Sigma C K)$, and in some cases the extension of infarction could not be detected with this technique.

In evaluating the quantification of ST segment elevation, however, the factors influencing the deviation of the ST segment on the praecordial leads must be taken into account. Though the origin of ST segment elevation, that is ischaemic injury current, is believed to be derived from the potential gradient across the boundary of ischaemia resulting from the alteration in action potential, electrical potential recorded on the chest surface can be influenced by other factors, for example the solid angle of the ischaemic zone subtended at a point of the electrode, and electrical conductivity of the tissues between the ischaemic zone and the electrode on the chest surface. Indeed, these factors which greatly influence underlying ischaemic potential gradient are of great importance in the interpretation of ST segment deviations in surface electrocardiograms, because the extent of infarction as well as the site of infarction directly influence the solid angle of the ischaemic area subtended at a point of the electrode. Even if individual variations in the potential gradient across the boundary of the ischaemic area and electrical conductivity of the thorax were both negligibly small, the difference in infarct site would greatly distort the distribution of ST segment elevation. In fact, in our study the regression lines indicating a significant correlation between $\Sigma S T$ or $n S T$ and infarct size $(\Sigma C K)$ in anterior infarction were quite different from those in inferior infarction. Moreover, in two patients who were excluded from the statistical analysis, one with lateral infarction and another with subendocardial infarction and only partial transmural anterior infarction, both $\Sigma S T$ and nST deviated downward from the regression line (see Fig. 6), indicating that in these cases the solid angle, which is proportional to ST segment elevation, was disproportionately small for the infarct size.

Similarly, infarct site can influence ST segment elevation in patients with inferior infarction. Indeed, there were two patients with inferior infarction like those with anterior infarction whose $\Sigma S T$ and nST substantially deviated from the 
regression line (see Fig. 7). Of these patients, one had an infarct extending towards the septal wall showing that $\Sigma S T$ and $\mathrm{nST}$ deviate upward from the regression line (Fig. 7). The other had an infarct extending toward the posterior wall, with downward deviation of $\Sigma S T$ and $\mathrm{nST}$ from the regression line (Fig. 7). In the former, the ST segment elevation caused by septal infarction might be superimposed on that of inferior infarction; while, in the latter, the reciprocal ST segment depression caused by posterior infarction may offset the underlying ST segment elevation. These results strongly suggest that both infarct site and infarct size are major factors which should be taken into account when we evaluate the ST segment elevation. In this study, apart from the patients with infarction extending toward other regions than the anterior or inferior wall and also in subendocardial infarction, the $\Sigma S T$ and $n S T$ correlated closely with the infarct size in patients both with anterior and with inferior infarction.

The present study showed that the time course of ST segment change was of great importance in assessing infarct size. Since the variation of measurement was so small as to be neglected (observation variation: $7 \cdot 6 \pm 8 \cdot 3 \%$ and hour-to-hour variation 48 hours after the onset: $3.5 \pm 2 \cdot 6 \%$ ), changes in ST segment elevation should reflect the change in extent and/or severity of ischaemia even though changes in electrical conductivity of the thorax such as that resulting from pulmonary oedema would also contribute to ST segment change to some extent. However, the extent of the ischaemic area may not be proportional to that of tissue necrosis, especially during the acute phase of infarction. Cox et al. (1968) showed that in experimental dogs the size of myocardial ischaemia reached its maximum 18 hours after ligation of a coronary artery, while that of myocardial necrosis reached its maximum several hours later than that of ischaemia. These findings agree with our present observation: ST segment elevation which should represent the extent and/or severity of ischaemia became maximal on average 35.2 hours after the onset of chest pain, while the duration of CK release, which represents the evolution of tissue necrosis, was on average about $\mathbf{4 0}$ hours.

The discrepancy of evolution between ischaemia and necrosis may be substantially large in the early phase of infarction because in this phase the regional oxygen balance is easily influenced by haemodynamic changes. Indeed, in more than $\mathbf{5 0}$ per cent of patients, $\Sigma S T$ and nST decreased a day after the onset while CK release continued. This discrepancy between the extent of ischaemia and necrosis size would account for poor correlation between $\Sigma$ ST or $\mathrm{nST}$ and infarct size ( $\Sigma \mathrm{CK}$ ) on admission and 12 hours after the onset of infarct. In contrast, ST segment elevation around the end of the evolution of infarct correlated highly with infarct size (Fig. 6 and 7). This finding indicates that at this stage the surrounding ischaemic area has already been established in proportion to infarct area.

Morris et al. (1974) also reported that the degree of ST segment displacement at $\mathbf{4 8}$ hours of infarction was related to the mean maximum serum AST (GOT) activity. This report agrees with our results. Poor or absent correlation between $\Sigma S T$ and infarct size ( $\Sigma C K)$ observed by Norris et al. (1976), and Thompson and Katavitis (1976) might have resulted because they measured $\Sigma S T$ long before the end of the evolution of infarction. The variation in infarct site in their studies might also account for poor correlation.

Although $\Sigma S T$ or $\mathrm{nST}$ at the end of the progression of the infarct or the maximal $\Sigma S T$ or $n S T$ can represent infarct size, the determination of these indices is difficult in patients who are not admitted immediately after the onset, and when enzymatic assessment of infarct size is not available. In such cases, praecordial ST segment mapping 48 hours after the onset could be of value in assessing infarct size.

The authors are grateful to Dr A. B. Weber for his help in the preparation of this manuscript and wish to thank Dr H. Nakao for his technical assistance.

\section{References}

Cox, J. L., McLaughlin, V. W., Flowers, N. C., and Horan, L. G. (1968). The ischemic zone surrounding acute myocardial infarction. Its morphology as detected by dehydrogenase staining. American Heart fournal, 76, 650-659.

Henning, H., Hardarson, T., Francis, G., O'Rourke, R. A., Ryan, W., and Ross, J., jun (1978). Approach to the estimation of myocardial infarct size by analysis of precordial S-T segment and R wave maps. American fournal of Cardiology, 41, 1-8.

Hori, M., Inoue, M., Fukui, S., Shimazu, T., Mishima, M., Ohgitani, N.,Minamino, T., and Abe, H. (1979). Correlation of ejection fraction and infarct size estimated from the total CK released in patients with acute myocardial infarction. British Heart fournal, 41, 433-440.

Inoue, M., Hori, M., Fukui, S., Abe, H., Minamino, T., Kodama, K., and Ohgitani, N. (1977). Evaluation of evolution of myocardial infarction by serial determinations of serum creatine kinase activity. British Heart fournal, 39, 485-492.

Maroko, P. R., Kjekshus, J. K., Sobel, B. E., Watanabe, T., Covell, J. W., Ross, J., jun, and Braunwald, E. 
(1971). Factors influencing infarct size following experimental coronary artery occlusions. Circulation, 43, 67-82.

Maroko, P. R., Libby, P., Covell, J. W., Sobel, B. E., Ross, J., jun, and Braunwald, E. (1972). Precordial S-T segment elevation mapping: an atraumatic method for assessing alterations in the extent of myocardial ischemic injury: the effects of pharmacologic and hemodynamic interventions. American fournal of Cardiology, 29, 223-230.

Mathey, D., Bleifeld, W., Hanrath, P., and Effert, S. (1974). Attempt to quantitate relation between cardiac function and infarct size in acute myocardial infarction. British Heart fournal, 36, 271-279.

Morris, G. K., Hayes, M. J., Hampton, J. R., and Mitchell, J. R. A. (1974). Predictive value of STsegment displacement and other indices after myocardial infarction. Lancet, 2, 372-374.

Muller, J. E., Maroko, P. R., and Braunwald, E. (1975). Evaluation of precordial electrocardiographic mapping as a means of assessing changes in myocardial ischemic injury. Circulation, 52, 16-27.

Norris, R. M., Barratt-Boyes, C., Heng, M. K., and Singh, B. N. (1976). Failure of ST segment elevation to predict severity of acute myocardial infarction. British Heart fournal, 38, 85-92.

Norris, R. M., Whitlock, R. M. L., Barratt-Boyes, C., and Small, C. W. (1975). Clinical measurement of myocardial infarct size: modification of a method for the estimation of total creatine phophokinase release after myocardial infarction. Circulation, 51, 614-620.

Page, D. L., Caulfield, J. B., Kastor, J. A., and DeSanctis, R. W. (1971). Myocardial changes associated with cardiogenic shock. New England fournal of Medicine, 285, 133-137.

Reese, L., Scheidt, S., and Killip, T. (1973). Variability of precordial ST segment maps after acute myocardial infarction in man (abstract). Circulation, 47 and 48, Suppl. IV, p. 38.

Sharpe, D. N., Botvinick, E. H., Shames, D. M., Norman, A., Chatterjee, K., and Parmley, W. W. (1978). The clinical estimation of acute myocardial infarct size with $99 \mathrm{~m}$-Technetium pyrophosphate scintigraphy. Circulation, 57, 307-313.

Sobel, B. E., Bresnahan, G. F., Shell, W. E., and Yoder, R. D. (1972). Estimation of infarct size in man and its relation to prognosis. Circulation, 46, 640-648.

Thompson, P. L., and Katavitis, V. (1976). Acute myocardial infarction-evaluation of praecordial ST segment mapping. British Heart fournal, 38, 10201024.

Requests for reprints to Dr Michitoshi Inoue, First Department of Medicine, Osaka University Medical School, 1-1-50 Fukushima, Fukushima-ku, Osaka 553, Japan. 